This item was submitted to Loughborough's Research Repository by the author.

Items in Figshare are protected by copyright, with all rights reserved, unless otherwise indicated.

\title{
Parameter estimation for VSI-fed PMSM based on a dynamic PSO with learning strategies
}

PLEASE CITE THE PUBLISHED VERSION

http://dx.doi.org/10.1109/TPEL.2016.2572186

PUBLISHER

(C) IEEE

VERSION

AM (Accepted Manuscript)

LICENCE

CC BY-NC-ND 4.0

\section{REPOSITORY RECORD}

Liu, Zhao-Hua, Hua-Liang Wei, Qing-Chang Zhong, Kan Liu, Xiao-Shi Xiao, and Liang-Hong Wu. 2019. "Parameter Estimation for Vsi-fed PMSM Based on a Dynamic PSO with Learning Strategies". figshare. https://hdl.handle.net/2134/22597. 


\title{
Parameter Estimation for VSI-Fed PMSM based on a Dynamic PSO with Learning Strategies
}

\author{
Zhao-Hua Liu ,Member, IEEE, Hua-Liang Wei, Qing-Chang Zhong, Senior Member, IEEE, Kan Liu, Member, \\ IEEE, Xiao-Shi Xiao ,and Liang-Hong Wu
}

\begin{abstract}
A dynamic particle swarm optimization with learning strategy (DPSO-LS) is proposed for key parameter estimation for permanent magnet synchronous machines (PMSMs), where the voltage-source-inverter (VSI) nonlinearities are taken into account in the parameter estimation model and can be estimated simultaneously with other machine parameters. In the DPSO-LS algorithm, a novel movement modification equation with variable exploration vector is designed to effectively update particles, enabling swarms to cover large areas of search space with large probability and thus the global search ability is enhanced. Moreover, a Gaussian-distribution based dynamic opposition-based learning (OBL) strategy is developed to help the pBest jump out local optima. The proposed DPSO-LS can significantly enhance the estimator model accuracy and dynamic performance. Finally, the proposed algorithm is applied to multiple parameter estimation including the VSI nonlinearities of a PMSM. The performance of DPSO-LS is compared with several existing PSO algorithms, and the comparison results show that the proposed parameters estimation method has better performance in tracking the variation of machine parameters effectively and estimating the VSI nonlinearities under different operation conditions.
\end{abstract}

Index Terms: particle swarm optimization (PSO), dynamic, opposition-based learning (OBL), learning strategy, system identification, parameter estimation, voltage source inverter (VSI) nonlinearity, permanent magnet synchronous machines (PMSMs).

\section{INTRODUCTION ${ }^{1}$}

$I^{N}$ $\mathrm{N}$ recent years, permanent magnet synchronous machines (PMSMs) are widely employed in high-performance applications (such as industrial robots, servo drive system, high-speed rail, renew energy generation systems, and household appliances) due to their high efficiency, high-power density, and good dynamic response [1][2]. Accurate knowledge of the machine parameters such as winding resistance, direct axis inductances, quadrate axis inductances, and rotor PM flux linkage are required for

Manuscript received January 18, 2016; revised April 1, 2016; accepted May 11,2016 . This work was supported in part by the National Natural Science Foundation of China under Grant (51374107,61503134,51577057, 61573299, 61403134), the China Postdoctoral Science Foundation funded project under Grant (2013M540628, 2014T70767), and the Hunan Provincial Education Department outstanding youth project under Grant (15B087).

Z.-H. Liu, X.-S. Xiao, and L.-H. Wu are with the School of Information and Electrical Engineering, Hunan University of Science and Technology, Xiangtan,411201,China.(e-mail:zhaohualiu2009@ @otmail.com).

H.-L.Wei is with the Department of Automatic Control and Systems Engineering, The University of Sheffield, Sheffield S1 3JD, U.K.(w.hualiang@sheffield.ac.uk).

Q.-C. Zhong is Department of Electrical and Computer Engineering, Illinois itute of Technology, Chicago, IL 60616, USA, (e-mail: zhongqc@ieee.org). K.Liu is with the Department of Electronic and Electrical Engineering, The University of Sheffield, Sheffield, S10 2TN, U.K. (email: 1kan@live.cn). control system design [3][4], condition monitoring and fault detection [5][6]. Usually machine parameters are used as a normal operation indicator for the PMSM drive system. For example, high temperature can cause an increase in winding resistance and the variation of inductances may cause torque ripple. Similarly, demagnetization in permanent magnet flux may cause a decrease in the amplitude of fundamental back electromotive force (EMF) and will influence $d$ - $q$-axis inductances of the machine, which in turn will have a direct impact on machines performance and power efficiency [7]. In real applications, these machine parameters are not known exactly as they vary with the load torque, the changes of temperature, and other environmental conditions. For example, the winding stator resistance is prone to change with the variation of temperature, and the rotor flux linkage created by PM may vary with the changes of temperature and/or operational condition [8] [9].

The PMSM parameters are a key factor for control system design, condition monitoring and fault diagnosis, and therefore estimation or direct measurement of the parameters are important. Traditionally, some measurement instruments such as thermal couplers, search coils and load test bench [10] [11] are employed to observe the machine parameters. However, there are some drawbacks in the direct measurement due to two reasons. Firstly, it is difficult to measure the machine parameters when PMSM operates; Secondly, even if direct measurement is possible, it would normally significantly increase the instruments cost and the complexity of the associated implementation. Some researchers proposed to use self-commissioning technique to estimate PMSM parameters under standstill state [12]. Unfortunately, this method cannot estimate all the needed parameters accurately on load, for example rotor PM flux linkage cannot be estimated at a standstill state. Thus, in practice system identification method is an ideal technology for directly estimating the needed parameters based on regularly measured signals instead of using additional measurement instruments [8]. Algorithms such as recursive least-squares (RLS) [13] [14], extended Kalman filter (EKF) [15] [16], model reference adaptive system (MRAS) [17] [18], finite-element method [19], adaptive estimation methods [20] and artificial neural networks (ANN) [21] are usually employed for the design of parameter estimators. Owing to linear parameterization for the RLS algorithm, the RLS estimator usually suffers from the noise characteristics and may lead to the reduction of solution accuracy [13]. In [15], an EKF was employed to estimate the winding resistance and rotor flux linkage. The results indicate that the estimator suffers from noise and instability, and thus cannot obtain accurate estimates for the actual parameter values. In [16], an EKF was proposed to estimate the rotor speed and position of 
PMSM. However, it is difficult to use in real applications because the algorithm is sensitive to noise. The MRAS estimators proposed in [17] and [18] cannot simultaneously estimate winding resistance, inductance and rotor flux linkage accurately. In the MRAS method, other parameters' nominal values specified in the motor manual are needed to estimate one parameter. In this case, the accuracy of identified parameters depends on the accuracy of the nominal value. In reality, the real parameter value usually depends on a variable operating condition caused by the changes of temperature and/or load. The FE (finite element) based parameters estimator proposed in [19] requires a high computational load and it may be challenging task to use this method to estimate PMSM parameters due to the complex computations. Some ANNs have also been proposed for estimating parameters of PMSM in [21]. Although the ANN method is known to be precise in parameter estimation, it usually needs a long data set to train and more knowledge for weight adjustment.

Some researchers proposed to inject perturbation signals into the drive system to obtain an extra number of state space equations of PMSM, and then and apply system identification methodologies to estimate machine parameters [8] [12] [22][23]. In the signal injection method, the designed estimator is based on the conventional $d$-q-axis equation and the VSI (voltage source inverter) nonlinearities are often ignored. Since the used signals such as VSI voltages for the parameter estimator are usually measured from the output voltage of the PI regulator in a PMSM vector control system, those methods are prone to suffer from the effect of nonlinearity of VSI such as switch voltage drop, switching delay and dead zone response [24]. As a result, it cannot estimate the actual value of the machine parameters due to the ignorance of effects of the nonlinearities on parameters estimation. Some methods were proposed to compensate the effect of VSI nonlinearity (see e.g. [24]-[27]), but these methods need accurate PMSM parameter values. Because the VSI nonlinearity and machine parameters cannot be simultaneously estimated by using these methods, the errors caused by VSI nonlinearity are combined into the parameter estimator and this will affect the accuracy of parameter estimation. Thus, the VSI nonlinearity should be considered in estimating machine parameters.

Due to the easy implementation, low computational cost and fast convergence speed in dealing with practical industrial problems, particle swarm optimization (PSO) has recently been introduced as an attractive optimization techniques in system identification, optimal and control of power electronics [28]-[34]. The parameter estimation problem can be treated as a grey-box model based parameter optimization task and the issue of parameter cross-coupling can be effectively solved by using PSO. In [28], an improved PSO method for estimating the unknown composite load model parameters was proposed, where a new crossover operation was introduced to improve the convergence performance. In [29], anther improved PSO was proposed for parameter estimation of an induction machine by modifying the movement equation of the standard PSO as linear time-varying parameters. A least mean square (LMS) method combined with PSO was presented to identify the parameters of an induction motor in [30]. In the field of PMSM parameters identification, some researchers proposed a PSO-based parameter estimator for PMSM [31] [32], this kind of intelligent estimator is effective in estimating the stator resistance and disturbed load torque. In [31], a PSO algorithm combined with experimental measurements was proposed for the identification of PMSMs. Similarly, a PSO combined with self-commissioning scheme was used in [32] for the identification and optimization of PMSM parameters. It is known that the basic PSO is easy to get trapped in local minima, these basic PSO algorithms may not be able to exactly estimate multiple parameters simultaneously, such as winding resistance, dq-axis inductances and permanent magnet flux. In [33], a coevolutionary optimization methodology combined multiple cooperate PSO and artificial immune system (AIS) was developed to improve multi-parameter estimation performance of PMSM. To speed up the search process of particles, a method of GPU accelerated parallel co-evolutionary immune PSO was proposed for parameter estimation and temperature monitoring of a PMSM [34], for which the performance of the parameter estimates was greatly improved by combining PSO and a parallel computing technology. Nevertheless, the existing PSO-based parameters estimators of PMSM are based on the basic $d$-q-axis equation which neglects the VSI nonlinearity, thus it cannot estimate the actual value of the machine parameters.

In this study, a dynamic particle swarm optimization with learning a strategy (DPSO-LS) is proposed to design the estimator of machine parameters and VSI nonlinearity in PMSM, in which the VSI nonlinearity is seen as a system parameter and can be estimated simultaneously with machine parameters. In the DPSO-LS, a novel movement modification equation with variable exploration vector is designed to update particles, it permits some particles with large probability to cover large areas of search space and thus the global search ability is enhanced. Moreover, a dynamic opposition-based learning (OBL) operator using Gaussian-distribution is developed to help pBest jump out local optima. OBL is a reinforcement learning strategy using computing and counter computing simultaneously, and is widely used to accelerate the convergence properties of many evolutionary algorithms [35][36]. The proposed algorithm is applied to the estimation of multiple machine parameters and VSI nonlinearity of PMSM. The results indicate that it can track parameter variation with the changing operation condition effectively. A comparison of the performance of DPSO-LS with several existing PSO algorithms is carried out, and it demonstrates that the proposed parameters estimation method can better track the variation of machine parameters and estimating the VSI nonlinearities under different operation conditions.

In summary, the major contributions of this paper include: 1) A dynamic estimator using dynamic PSO combined with a dynamic OBL scheme is proposed for simultaneous estimation of the machine parameters and VSI nonlinearities of PMSM. The VSI nonlinearity is seen as system parameters and can be estimated by the designed estimator, which takes into consideration of the influence of distorted voltage from the VSI and thus the accuracy of the estimation of machine parameters is improved.The proposed estimator can 
simultaneously estimate machine parameters and VSI nonlinearity without any priori knowledge of the PMSM.

2) In order to enhance the accuracy and the dynamic performance of the estimator, a novel movement modification equation using a variable exploration vector is designed to update the velocity of particles. Moreover, a dynamic OBL mechanism with Gaussian distribution is introduced to overcome the blindness of the pBest stochastic evolution and enables it jump out the local optimality. The proposed estimator has a fast convergence rate, a good accuracy and a good dynamic response property.

3) The proposed estimator has also taken into account the identifiability of all the parameters to be estimated and the VSI nonlinearities. Furthermore VSI nonlinearities can be estimated individually from the $d$ - $q$-axis equation with $i d=0$ control and $i d \neq 0$ (current injection condition), respectively.

The remainder of this paper is organized as follows. An estimator model for the machine parameters and VSI nonlinearities of a PMSM is described in section II. A DPSO-LS algorithm is proposed in section III, where a scheme to determine the parameters of the estimator and the optimization procedure and steps are described. Experimental results and the analysis are given in section IV. Finally, some concluding remarks are presented in section $\mathrm{V}$.

\section{PMSM MODEL AND DESIGN OF PARAMETER ESTIMATION MODEL}

\section{A. PMSM Model and VSI Nonlinearity}

The $d$ - $q$-axis voltage equations of the PMSM are usually employed for the parameter estimation of the machine [8], which are given as:

$$
\begin{aligned}
& \left\{\begin{array}{l}
\frac{d i_{d}}{d t}=-\frac{R}{L_{d}} i_{d}+\frac{L_{q}}{L_{d}} \omega i_{q}+\frac{u_{d}}{L_{d}} \\
\frac{d i_{q}}{d t}=-\frac{R}{L_{q}} i_{q}-\frac{L_{d}}{L_{q}} \omega i_{d}+\frac{u_{q}}{L_{q}}-\frac{\psi}{L_{q}} \omega
\end{array}\right. \\
& T_{e}=1.5 P\left[\psi i_{q}+\left(L_{d}-L_{q}\right) i_{d} i_{q}\right]
\end{aligned}
$$

where $\omega$ is the electrical angular velocity, $u_{d}, u_{q} i_{d}$, and $i_{q}$, are $d$ - $q$-axis stator voltage and current, $P$ is the number of pole pairs ,the parameter $\operatorname{set}\left\{R, L_{d}, L_{q}, \psi\right\}$ is unknown and needs to be identified from measured data. (1) can be discretized as follows when the machine is under the steady-state:

$\left\{\begin{array}{l}u_{d}(k)=R i_{d}(k)-L_{q} \omega(k) i_{q}(k) \\ u_{q}(k)=R i_{q}(k)+L_{d} \omega(k) i_{d}(k)+\psi \omega(k)\end{array}\right.$

Taking into account the influence of VSI nonlinearity, (3) could be rewritten as:

$$
\left\{\begin{array}{l}
\dot{u}_{d}(k)+D d(k) V_{\text {dead }}=u_{d}(k) \\
\dot{u}_{q}(k)+D q(k) V_{\text {dead }}=u_{q}(k)
\end{array}\right.
$$

where $\dot{u}_{d}$ and $\dot{u}_{q}$ are the $d q$-axis reference voltages and measured from the PI regulators (V), $D d$ and $D q$ can be expressed as [25]-[27]:

$$
\left[\begin{array}{l}
D d(k) \\
D q(k)
\end{array}\right]=2\left[\begin{array}{ccc}
\cos (\theta) & \cos \left(\theta-\frac{2 \pi}{3}\right) & \cos \left(\theta+\frac{2 \pi}{3}\right) \\
-\sin (\theta) & -\sin \left(\theta-\frac{2 \pi}{3}\right) & \sin \left(\theta-\frac{\pi}{3}\right)
\end{array}\right]\left[\begin{array}{l}
\operatorname{sign}\left(i_{a s}\right) \\
\operatorname{sign}\left(i_{b s}\right) \\
\operatorname{sign}\left(i_{c s}\right)
\end{array}\right]
$$

where $i_{a s}, i_{b s}, i_{c s}$ are the stator $a b c$ three-phase currents (A).

$\operatorname{sign}= \begin{cases}1, & , i \geq 0 \\ -1, & i<0\end{cases}$

In discrete time domain, the steady-state PMSM equation including the distorted voltage due to VSI nonlinearity can be expressed as

$$
\begin{aligned}
& \dot{u}_{d}(k)+D d(k) V_{\text {dead }}=R i_{d}(k)-L_{q} \omega(k) i_{q}(k) \\
& \dot{u}_{q}(k)+D q(k) V_{\text {dead }}=R i_{q}(k)+L_{d} \omega(k) i_{d}(k)+\psi \omega(k)(7 \mathrm{~b})
\end{aligned}
$$

The variable $V_{\text {dead }}$ is the distorted voltage caused by the VSI nonlinearities, and can be represented as

$$
V_{\text {dead }}=\frac{T_{\text {dead }}+T_{\text {on }}-T_{\text {off }}}{T s} \cdot\left(V_{d c}-V_{\text {sat }}+V_{d}\right)+\frac{V_{\text {sat }}+V_{d}}{2}
$$

where $T_{\text {dead }}, T_{\text {on }}, T_{\text {off }}, V_{d c}, V_{\text {sat }}$ and $V_{d}$ are the dead-time period, turn-on ,turn-off times of the switching device, the actual and measured real-time dc bus voltages, the saturation voltage drop of the active switch and the forward voltage drop of the freewheeling diode, respectively. The switching times and voltage drops in the switching device may vary during operations and difficult to measure due to the changes of current, frequency, DC link voltage, operating conditions , dead-time period, and temperature. Therefore, it is important to accurately estimate and compensate the $V_{\text {dead }}$ in order to ensure the accuracy of estimated PMSM parameter values.

\section{B. PMSM Parameter Estimator Design}

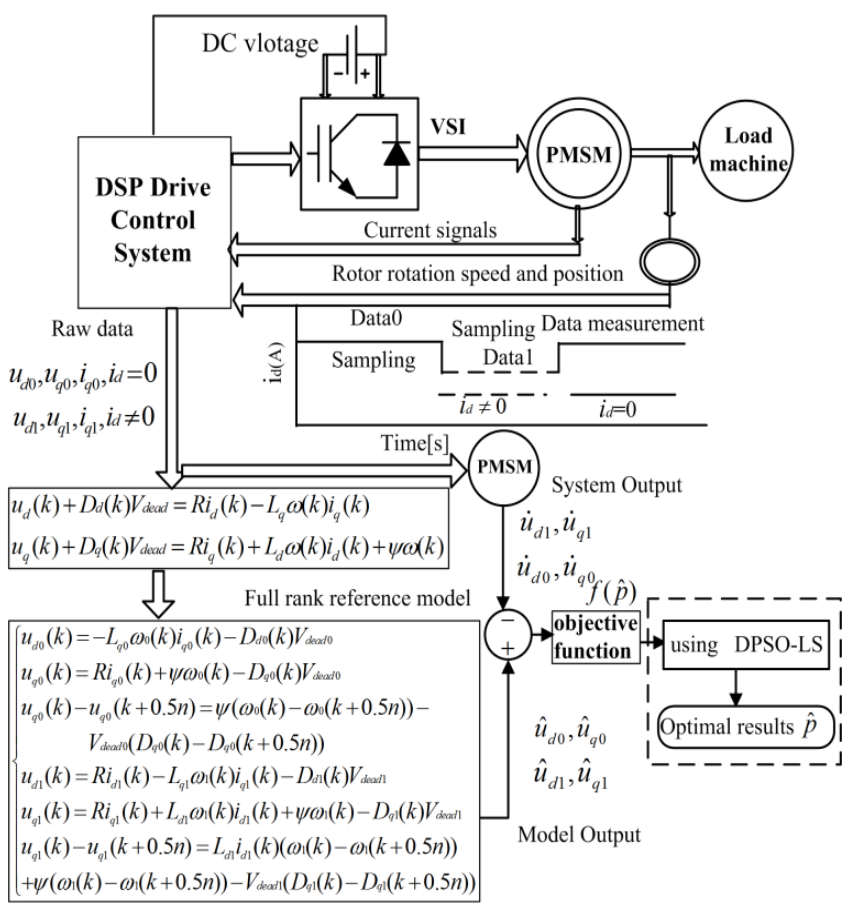

Fig .1.Schematic diagrams of estimation and mathematical model. 
First of all, the VSI nonlinearity is seen as parameters that can be estimated simultaneously with other machine parameters by the designed estimator using the proposed DPSO-LS algorithm. To obtain more equations, $i_{d}$ is set to be zero for decoupling the flux and torque control, and a very short period of negative $i_{d}$ (i.e. $i_{d}<0$ ) is then injected to obtain a full rank reference model. The symbols $i d_{0}$ and $i d_{l}$ indicate that the $d$ axis is injected with the current $i d=0$ and $i d \neq 0$, respectively (see Fig. 1). In Fig.1, under normal operation condition, the PMSM is under a control state with $i d=0$, the Data0 (the data associated with $i_{d}=0$ ) are firstly measured and then a short pulse of negative $i d<0$ is injected, and the corresponding data, Data1 (the data associated with $i d \neq 0$ ), are recorded after $2 \mathrm{~ms}$ of injection, both with the same sampling width and sampling number.

The parameters of machine are assumed to be constant as the duration of injected pulse current is very short due to mechanical inertia and fast response of current loop PI controller. In this case, the influence of injecting a short pulse of $i d$ on output torque can be neglected. Thus, the two sets of steady state data (Data0 and Data1) can be used for the estimation of multiple machine parameters since these parameters can be seen as constant within very short time. Note that $V_{\text {dead }}$ is directly related to the current, so $V_{\text {dead } 0} \neq V_{\text {dead } 1}$. Thus, the distorted voltage $V_{\text {dead0 } 0}$ and $V_{\text {dead } 1}$ need to be estimated from the data. A total of six parameters (i.e., $\left.R, L_{d}, L_{q}, \psi, V_{\text {dead } 0}, V_{\text {dead } 1}\right)$ need to be estimated, therefore six equations need to be designed. From (7), it is ready to get (9a) and (9b) when $i d=0$. When $i d \neq 0$, (7) can be reformed as (9d) and (9e). The measured data are divided into two parts, the first part is indexed from 1 to $0.5 n$ and the second one is indexed from $0.5 n+1$ to $n$. Since $i q$ and $i d$ are kept as constants, it is reasonable that $V_{\text {dead }}$ is regarded as a constant during the data measurement, two additional equations are designed as in (9c) and (9f) during the varying speed. The full rank reference model is given as

$$
\begin{aligned}
& u_{d 0}(k)=-L_{q 0} \omega_{0}(k) i_{q 0}(k)-D_{d 0}(k) V_{\text {dead } 0} \\
& u_{q 0}(k)= R i_{q 0}(k)+\psi \omega_{0}(k)-D_{q 0}(k) V_{\text {dead } 0} \\
& u_{q 0}(k)-u_{q 0}(k+0.5 n)=\psi\left(\omega_{0}(k)-\omega_{0}(k+0.5 n)\right)- \quad V_{\text {dead } 0}\left(D_{q 0}(k)-D_{q 0}(k+0.5 n)\right) \\
& u_{d 1}(k)= R i_{d 1}(k)-L_{q 1} \omega_{1}(k) i_{q 1}(k)-D_{d 0}(k) V_{\text {dead } 1} \\
& u_{q 1}(k)= R i_{q 1}(k)+L_{d 1} \omega_{1}(k) i_{d 1}(k)+\psi \omega_{1}(k)-D_{q 1}(k) V_{\text {dead } 1} \\
& u_{q 1}(k)-u_{q 1}(k+0.5 n)=L_{d} i_{d 1}(k)\left(\omega_{1}(k)-\omega_{1}(k+0.5 n)\right) \\
&+\psi\left(\omega_{1}(k)-\omega_{1}(k+0.5 n)\right)-V_{\text {dead } 1}\left(D q_{1}(k)-D q_{1}(k+0.5 n)\right)
\end{aligned}
$$

The estimation of the parameters can be addressed as an optimization problem where the system response to a known input is used to find the unknown parameter values of the model. The idea is to compare the system response with the parameterized model based on a cost function, which is defined to measure the similarity between the system response and the model response.

To approximate the dynamic parameters, it needs to define cost functions. In this study, the three cost functions or fitness functions, under control $i d=0$, are defined as

$$
\begin{aligned}
& f_{1}\left(\hat{L}_{q}, \hat{V}_{\text {dead } 0}\right)=\frac{1}{n} \sum_{k=1}^{n}\left|\dot{u}_{d 0}(k)+D_{d 0}(k) \hat{V}_{\text {dead } 0}-\hat{u}_{d 0}(k)\right| \\
& =\frac{1}{n} \sum_{k=1}^{n}\left|\dot{u}_{d 0}(k)+D_{d 0}(k) \hat{V}_{\text {dead } 0}+\omega_{0}(k) \hat{L}_{q} i_{q 0}(k)\right| \\
& f_{2}\left(\hat{R}, \hat{\psi}, \hat{V}_{\text {dead } 0}\right)=\frac{1}{n} \sum_{k=1}^{n}\left|\dot{u}_{q 0}(k)+D_{q 0}(k) \hat{V}_{\text {dead } 0}-\hat{u}_{q 0}(k)\right| \\
& =\frac{1}{n} \sum_{k=1}^{n}\left|\dot{u}_{q 0}(k)+D_{q 0}(k) \hat{V}_{\text {dead } 0}-\hat{R} i_{q 0}(k)-\omega_{0}(k) \hat{\psi}\right| \\
& f_{3}\left(\hat{\psi}, \hat{V}_{\text {dead } 0}\right)=\frac{1}{2 n} \sum_{k=1}^{0.5 n} \mid \dot{u}_{q 0}(k)-\dot{U}_{q 0}(k+0.5 n)-\left(\hat{\psi}\left(\omega_{00}(k)-\omega_{0}(k+0.5 n)\right) \mid\right.
\end{aligned}
$$

Under control $i d \neq 0$, another three fitness functions are given as

$$
\begin{gathered}
f_{4}\left(\hat{R}, \hat{L}_{q}, \hat{V}_{\text {dead } 1}\right)=\frac{1}{n} \sum_{k=1}^{n}\left|\dot{u}_{d 1}(k)+D d_{1}(k) \hat{V}_{\text {dead } 1}-\hat{u}_{d 1}(k)\right| \\
=\frac{1}{n} \sum_{k=1}^{n}\left|\dot{u}_{d 1}(k)+D_{d 1}(k) \hat{V}_{\text {dead } 1}-\hat{R} i_{d 1}(k)+\omega_{1}(k) \hat{L}_{q 1} i_{q 1}(k)\right| \\
f_{5}\left(\hat{R}, \hat{\psi}, \hat{L}_{d}, V_{\text {dead } 1}\right)=\frac{1}{n} \sum_{k=1}^{n}\left|\dot{u}_{q 1}(k)+D_{q 1}(k) \hat{V}_{\text {dead } 1}-\hat{u}_{q 1}(k)\right| \\
=\frac{1}{n} \sum_{k=1}^{n}\left|\begin{array}{l}
\dot{u}_{q}(k)+D_{q 1}(k) \hat{V}_{\text {dead } 1}-\omega_{1}(k) \hat{L}_{d 1}(k) i_{d}(k) \mid \\
-\hat{R} i_{q 1}(k)-\omega_{1}(k) \hat{\psi}(k)
\end{array}\right| \\
f_{6}\left(\hat{R}, \hat{\psi}, \hat{V}_{\text {dead } 1}\right)=\frac{1}{2 n} \sum_{k=1}^{0.5 n}\left|\begin{array}{l}
\dot{u}_{q 1}(k)-\dot{u}_{q 1}(k+0.5 n)- \\
\hat{L}_{d} i_{d 1}(k)\left(\omega_{1}(k)-\omega_{1}(k+0.5 n)\right) \\
+\hat{\psi}\left(\omega_{1}(k)-\omega_{1}(k+0.5 n)\right)- \\
\left.\hat{V}_{\text {dead } 1}\left(D q_{1}(k)-D q_{1}(k+0.5 n)\right)\right)
\end{array}\right|
\end{gathered}
$$

where $n$ is the length of samples, $\hat{u}_{d}$ and $\hat{u}_{q}$ indicate the estimated voltages in $d q$-axis calculated through measured currents and the estimated parameters and VSI nonlinearities. Let $\hat{p}=\left(\hat{R}, \hat{L}_{d}, \hat{L_{q}}, \hat{\psi}, V_{\text {dead }}, V_{\text {dead } 1}\right)$, then all the needed parameters can be identified simultaneously by minimizing the following objective function

$$
f(\hat{p})=\sum_{i=1}^{6} a_{i} f_{i}
$$

where $a_{i} s$ are weighting coefficients. Note that the designed objective function (16) is related to the actual permanent magnet synchronous motor drive system which is highly nonlinear and time varying. The nonlinear objective function has many local optima and the used optimization method should be capable of identifying parameters dynamically and adaptively in solving such a problem. So, it is important to develop an efficient dynamic parameter tracking approach for the estimation of PMSM parameters and VSI nonlinearities.

\section{ESTIMATOR PARAMETER OPTIMIZATION WITH DPSO-LS}

\section{A. Principle of the Basic PSO Algorithm}

PSO is a swarm-based intelligent optimization algorithm inspired by the ideas of mimicking behaviors of bird flocking foraging. Assuming that the problem is defined in a $d$-dimensional space, and each particle $i$ is composed of two vectors, namely the velocity vector $V_{\mathrm{i}}=\left\{V_{\mathrm{i} 1}, V_{\mathrm{i} 2}, \ldots, V_{\mathrm{id}}\right\}$ and the position vector $X_{\mathrm{i}}=\left\{X_{\mathrm{i} 1}, X_{\mathrm{i} 2}, \ldots, X_{\mathrm{id}}\right\}$, the searching scheme can be represented as 


$$
\begin{aligned}
V_{i d}(t+1)= & \phi V_{i d}+c_{1} * \operatorname{rand}_{1}()\left(\text { pBest }_{i d}(t)-X_{i d}(t)\right) \\
& +c_{2} * \operatorname{rand}_{2}()\left(\text { gBest }_{d}(t)-X_{i d}(t)\right) \\
X_{i d}(t+1)= & X_{i d}(t)+V_{i d}(t+1)
\end{aligned}
$$

where $\phi$ is the inertia weight factor decreasing linearly, $c_{1}$ and $c_{2}$ are the acceleration coefficients, rand $_{1}$ and rand $_{2}$ are two uniformly distributed numbers generated randomly within [0,1], respectively. pBest $i_{i d}$ represents the $i$-th particle has found best position so far (individual best), gBest $_{d}$ is the best position found among the entire population (global best).

\section{B. The Proposed DPSO-LS Algorithm}

As mentioned in Section II, the objective function is multimodal and therefore requires that the proposed parameter optimization should be able to adaptively change their original trajectories to explore new search space when the solution of problem is changing. In order to solve this problem, a dynamic particle swarm optimization with a learning strategy (DPSO-LS) is proposed for machine parameter and VSI nonlinearity estimation.

The implementation of the learning strategy contains two key aspects. Firstly, a novel movement update equation is designed using a variable exploration vector to enhance the dynamic performance of PSO. Secondly, a dynamic $O B L$ using adaptive Gaussian-distribution is proposed to overcome the blindness in the search of pBests through stochastic evolution and enables it escape from local optima. It worth noting that the proposed DPSO-LS does not increase time complexity in comparison with the basic PSO. Actually, it is easy to implement to solve the optimization problem here, and to adapt to solve other similar problems.

The general steps of DPSO-LS are stated as follows.

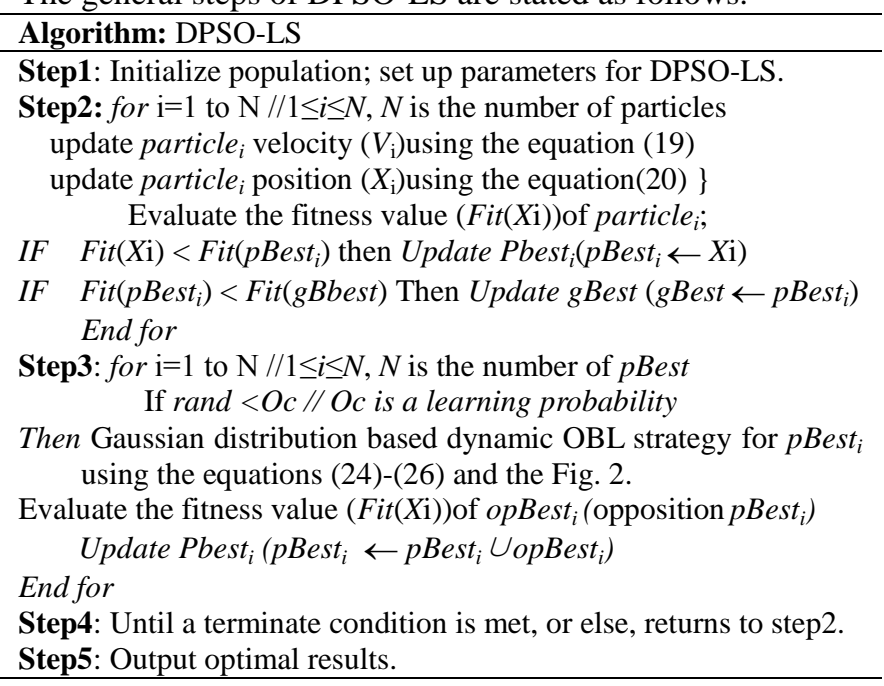

\section{Dynamic PSO Model}

In recent years, many researchers focused on improving the performance of basic PSO [33] [34] (mainly on individual best position (pBest) and the global best position ( $g$ Best)). If the particles converge fast, they will always shrink toward local regions within a few generations [33]. This phenomenon leads to a similar search behavior among the swarms and the loss of diversity in the population. If the particles are trapped in local regions, they will not be able to jump out due to their homogeneous search behavior as well as the absence of an adaptive exploration ability [33].To improve the performance of PSO, the particles should be able to adaptively change their original trajectories to explore new search space. The issue is how to guide particles to move toward different promising regions and enhance a broader exploration of the solution space. An improved movement modification equation with an adaptive exploration vector is proposed to update the velocity of particles ,i.e.,

$$
\begin{gathered}
V_{i d}(t+1)=\phi V_{i d}+c_{1} * \operatorname{rand}_{1}()\left(\operatorname{Pbest}(t)-X_{i d}(t)\right) \\
+c_{2} * \operatorname{rand}_{2}()\left(\operatorname{gBest}(t)-X_{i d}(t)\right) \\
+c_{3} * \operatorname{rand}_{3}()\left(R(t)-X_{i d}(t)\right) \\
X_{i d}(t+1)=X_{i d}(t)+V_{i d}(t+1)
\end{gathered}
$$

The exploration vector $\left(R(t)-X_{j d}(t)\right)$ is added to provide a broader exploration of the solution space for the $i$-th particle due to the use of adaptive variable exploration radius $(R(t))$ which permits particles to cover large areas of search space with large probability.

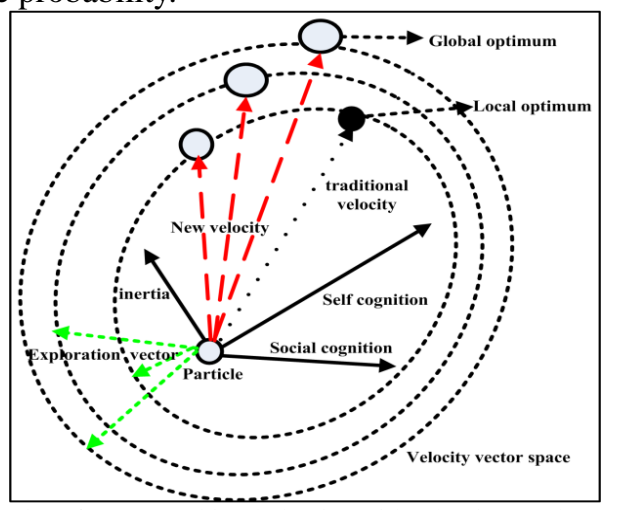

Fig.2.IllustrationofPSO searching behavior with adaptive explore mechanism

The exploration radius $R(t)$ learns adaptively as

$$
R(t)=\frac{\left(X_{\max }^{d}+X_{\min }^{d}\right)}{2}+\frac{\left(X_{\max }^{d}-X_{\min }^{d}\right)}{2} \cdot e^{-\lambda t} \cdot \cos (2 \pi u)(21)
$$

where $u$ is a random in $[0,1], X_{\text {min }}^{d}$ and $X_{\text {max }}^{d}$ are the designated lower and upper bounds of the problem, respectively, $\lambda$ is an adjustment parameter ( $\lambda$ is usually $\geqslant$ 2 ), and $t$ is the iteration index. The modified velocity equation of PSO indicates that the swarm members are allowed to explore larger unvisited regions in the objective space (as illustrated in Fig.2). A large $R(t)$ facilitates an exploration which induces the particles to leave their current regions and pushes the particles to search in the other regions. A small $R(t)$ enhances the exploitation which refines the best solution of the particles by exploiting a small vicinity around this best solution. Obviously, the whole feasible solution space can be explored and covered as large as possible by this modified equation with adaptive variable exploration vector.

\section{A Dynamic OBL for pBests}

In order to help pBest particles jump out the local optimal and accelerate the local search, in this study an adaptive Gaussian-distributed-based dynamic OBL strategy is proposed for a reinforcement learning of pBest. 


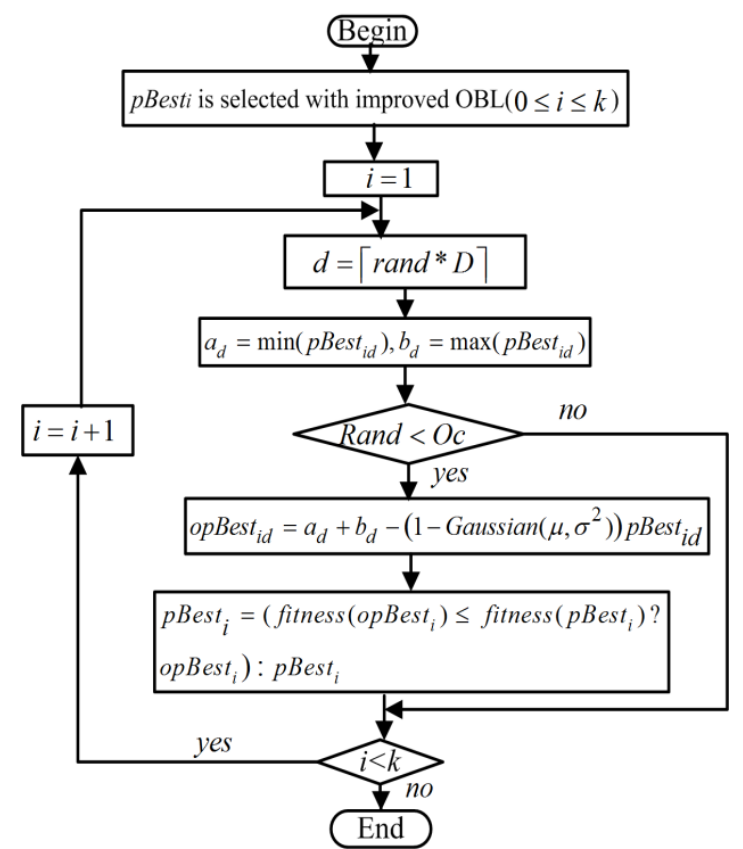

Fig.3. The schematic of dynamic OBL for pBests.

The opposition-based learning (OBL) is a machine learning method and was firstly introduced by Tizhoosh [35].

The key point for OBL is that it introduces a simple technique which allows the population-based algorithms to search for an optimal point in the opposite direction of the current search. Mathematical and experimental proofs show that opposite points are more beneficial than random points [36], and can be used to accelerate the convergence properties of other evolutionary algorithms. The basic idea of OBL is that a search in the opposite direction is carried out simultaneously when a solution is exploited in a direction, i.e.,

$$
\tilde{x}=a+b-x
$$

where $x$ is a real number on the interval $[a, b]$, and $\tilde{x}$ is the opposite number of $x$. This definition is also valid for D-dimensional space, where for $x_{1}, x_{2}, \ldots, x_{\mathrm{D}} \in R$ and $x_{\mathrm{i}} \in\left[a_{\mathrm{i}}, b_{\mathrm{i}}\right]$, the D-dimensional point $x_{\mathrm{i}}$ cis defined as

$$
\tilde{x}_{i}=a_{i}+b_{i}-x_{i}
$$

In order to overcome the drawbacks of the traditional $O B L$ and enhance the pBest convergence speed, in this study a dynamic OBL strategy using adaptive Gaussian distribution is designed as

$$
\begin{aligned}
& \text { opBest }_{i d}=a_{d}(t)+b_{d}(t)-\left(1-\operatorname{Gaussian}\left(\mu, \sigma^{2}\right)\right) \cdot p \text { Best }_{i d} \\
& a_{d}(t)=\min \left(p \text { Best }_{i d}\right), b_{d}(t)=\max \left(p \text { Best }_{i d}\right)
\end{aligned}
$$

where $\operatorname{Gaussian}\left(\mu, \sigma^{2}\right)$ is a random number of a Gaussian distribution with a zero mean $(\mu)$ and a standard deviation $(\sigma)$. In order to obtain a better dynamic learning performance for $p$ Bests, it is assumed that $\sigma$ decreases nonlinearly, for which a good choice may be given as

$$
\sigma=\sigma_{\min }+\left(\sigma_{\max }-\sigma_{\min }\right)\left(1-\frac{t}{T}\right)^{2}
$$

where $\sigma_{\max }$ (fixed to one in this study) and $\sigma_{\min }$ (fixed to zero)are the upper and lower bounds of $\sigma$, which specifies the learning scale to reach a new region. We adopt the
Box-Muller transform [37] to obtain a Gaussian distributed random variable, i.e.,

$$
\operatorname{Gaussian}\left(\mu, \sigma^{2}\right)=\mu+\sigma \sqrt{-2 \ln \left(1-u_{1}\right)} \cdot \cos \left(2 \pi \cdot u_{2}\right)
$$

where $u_{1}$ and $u_{2}$ are uniformly generated random numbers in $[0,1]$.

The schematic of the dynamic OBL for pBests is shown in Fig.3, where the symbol $O c$ is the learning probability, the symbol $d$ is the randomly selected from total dimension $(D)$ as $d=\left\lfloor\right.$ rand $\left.^{*} D\right\rfloor$. Since not all dimensions are changed to their opposite values during OBL, useful information in the original individual may be preserved. The point and its opposite point are evaluated simultaneously in order to continue with the one that best fit, that is, if the fitness \left.${\text { ( } \text { pBest }_{i}}^{\text {new }}\right)$ is better than fitness $\left(p B e s t_{i}\right)$, then the pBest position of $i$-th particle will be replaced with opBest $t_{\mathrm{i}}$; otherwise, we continue with pBest $t_{\mathrm{i}}$. From the above description, it is quite clear that the pBest can be forced to jump to a new solution candidate with the improved $O B L$ technique. This strategy provides a disturbance at $p B e s t$, the jump out performance is enhanced by this improved $O B L$ with dynamic Gaussian distribution which is beneficial to guide particles' moving direction and enhance convergence speed.

\section{EXPERIMENTAL RESULTS}

\section{A. Hardware Control System and Software Platform}

The parameter estimation process is divided into two main procedures, namely, the experimental data acquisition and data processing. To perform our experiments, a permanent magnet synchronous motor prototype and DSP vector control hardware platform are used as the experimental facility which is listed in Fig.4 and Table I. The current signals are obtained from the Hall transducers and then sampled by the DSP. The DC link is connected with the DC power source whose output is fixed to $36 \mathrm{~V}$. The sampling period is set to $83.3^{\mu} \mathrm{s}$. The signals from the DSP are transmitted to a PC via serial protocol communication network and recorded in memory for

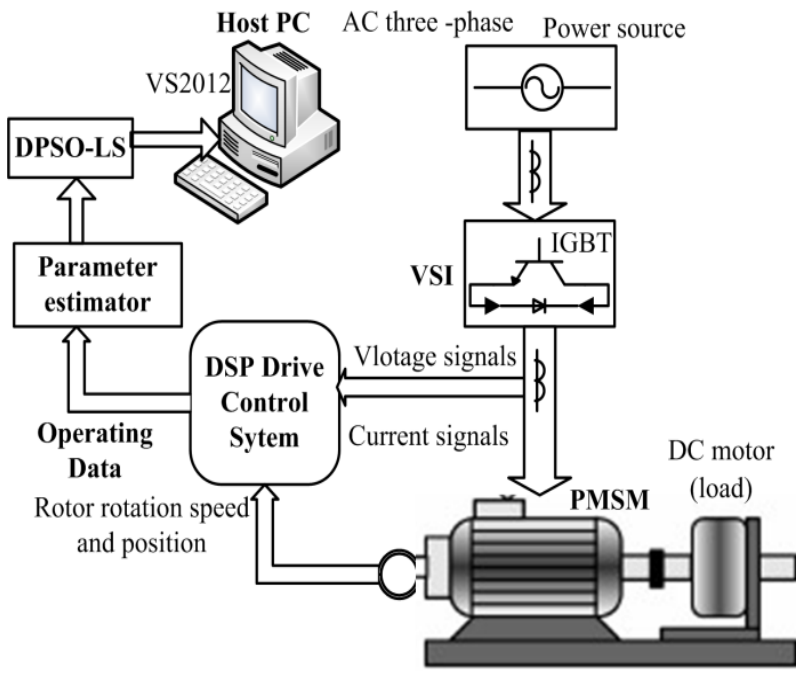

Fig.4.The schematic diagram of hardware and software platform for system identification and parameter estimation in PMSM 
late use of parameter estimation using the proposed DPSO-LS algorithm, and this is carried out in a host computer installed with visual studio 2012 software.

A series of hybrid PSOs are used for a comparison with DPSO-LS. The hybrid PSOs algorithms used include OPSO (OBL for PSO) [35], HGAPSO (hybrid PSO with genetic algorithm) [38], HPSOWM (hybrid PSO with Wavelet Mutation) [39], CLPSO (comprehensive learning PSO) [40], A-CLPSO (An improved comprehensive learning PSO) [41] and APSO (adaptive Particle Swarm Optimization) [42], which are frequently used to test the performance of newly developed algorithms for dynamic optimization problems. To assess the quality of the estimated machine parameters and VSI nonlinearities based on the proposed DPSO-LS, a statistical analysis is performed and the associated mean, standard deviation and $t$-test values are calculated.

With the consideration of the balance between time cost and the precision of solutions, in this study the optimal setting of parameters for the proposed DPSO-LS is recommended as follows: the population size is 50 , the inertia weight in (19) is on $[0.90,0.4]$ and decreases linearly, the two acceleration coefficients c1, c2 are 1.49445 , the adjustment parameter $\lambda$ set to 6 in equation (12), and the opposition learning probability $(O c)$ is set to 0.38 in Fig.3. The optimal settings for other hybrid PSOs are the same as suggested in the associated references.

The basic settings of these PSOs are as follows: all the PSO algorithms using the same population size of 50 , the maximum iteration is 300 and the number of runs is 30. All the hybrid PSOs are operated on the same hardware and software platform. All experiments are carried out on the same computer equipped with Intel $^{\circledR}{ }^{\circledR}$-core $^{\mathrm{TM}}-\mathrm{i} 5-2450 \mathrm{M}$ and 4.0GB DDR3 RAM.

TABLE I

DESIGN PARAMETERS AND SPECIFICATION OF PMSM

\begin{tabular}{cc}
\hline Rated speed & $400 \mathrm{rpm}$ \\
\hline Rated current & $4 \mathrm{~A}$ \\
\hline DC link voltage & $36 \mathrm{v}$ \\
Nominal terminal wire resistance & 0.043 \\
Nominal self inductance & $2.91 \mathrm{mh}$ \\
Nominal mutual inductance & $-0.330 \mathrm{mh}$ \\
Nominal $d$-axis inductance & $3.24 \mathrm{mh}$ \\
Nominal $q$-axis inductance & $3.24 \mathrm{mh}$ \\
Nominal amplitude of flux induced by magnets & $77.6 \mathrm{mWb}$ \\
Number of pole pairs & 5 \\
Nominal phase resistance $\left(T=25{ }^{\circ} \mathrm{C}\right)$ & $0.330 \Omega$ \\
Inertia & $0.8 \mathrm{e}-5 \mathrm{kgm}^{2}$ \\
\hline
\end{tabular}

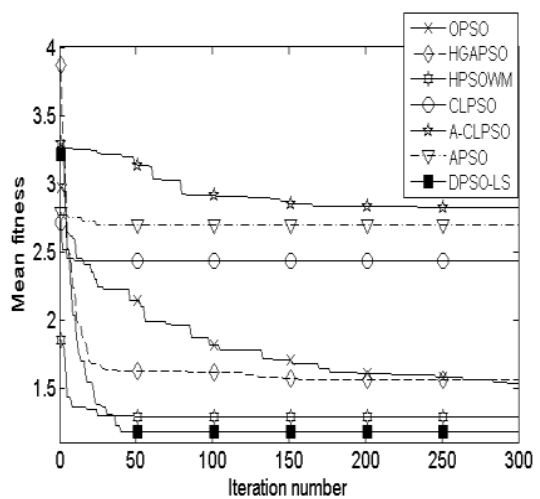

Fig.5. The fitness convergence curve of seven PSOs on multiple parameters and VSI nonlinearities identification of PMSM under normal temperature.

\section{B. Estimation of PMSM Parameters and VSI Nonlinearities under Normal Temperature}

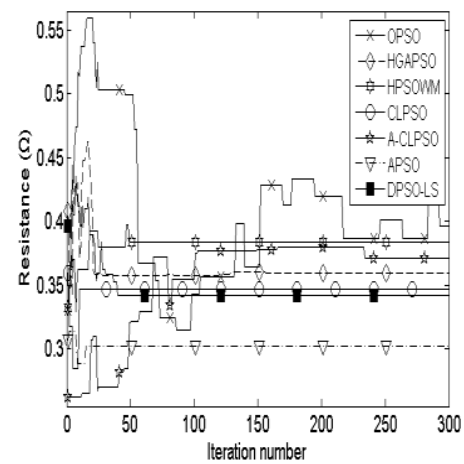

(a)

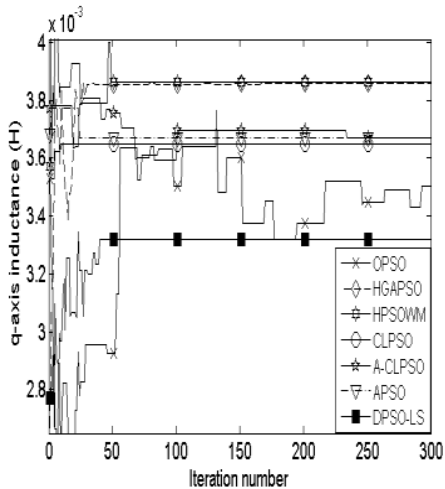

(d)

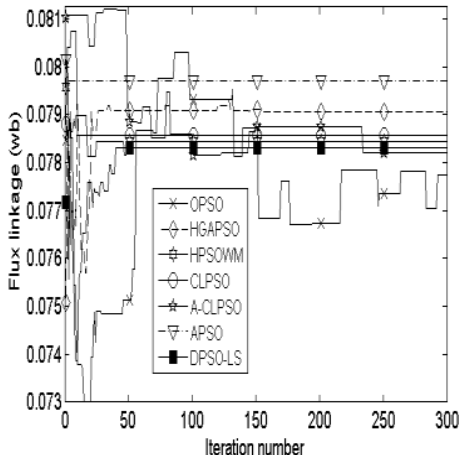

(b)

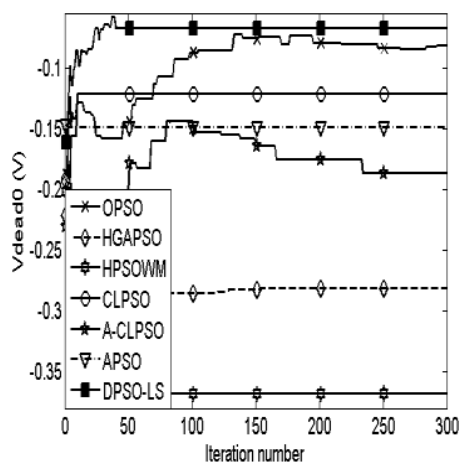

(e)

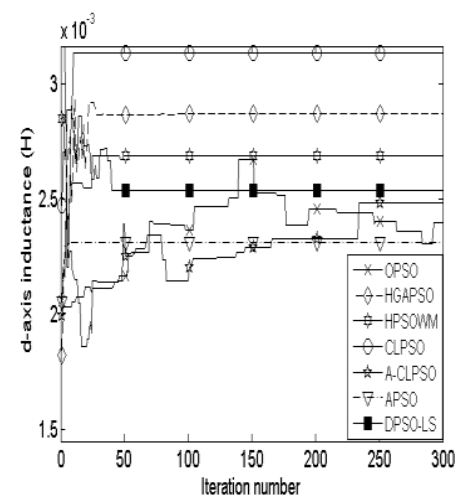

(c)

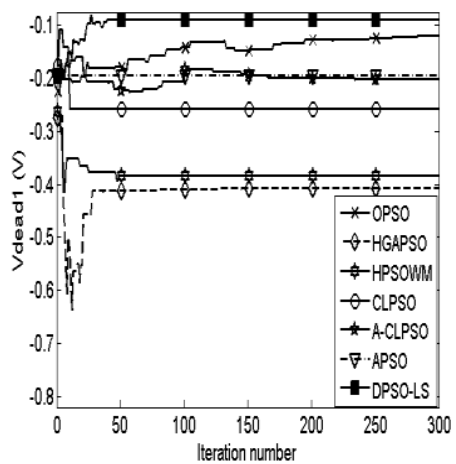

(f)

Fig.6.Identified parameters under normal temperature. (a) winding resistance. (b) rotor flux linkage (c) $d$-axis inductance.(d) $q$-axis inductance.(e) $V_{\text {dead0 }}$.(f) $V_{\text {dead1 }}$. 
TABLE II.

RESULT COMPARISONS AMONG SEVEN PSOS ON MULTIPLE PARAMETERS AND VSI NONLINEARITIES IDENTIFICATION OF PMSM UNDER NORMAL TEMPERATURE.

\begin{tabular}{|c|c|c|c|c|c|c|c|}
\hline Estimated Parameters & OPSO & HGAPSO & HPSOWM & CLPSO & A-CLPSO & APSO & DPSO-LS \\
\hline$R(\Omega)$ & 0.396 & 0.359 & 0.384 & 0.346 & 0.371 & 0.302 & 0.342 \\
\hline$\psi(w b)$ & 0.0777 & 0.0790 & 0.0784 & 0.0785 & 0.782 & 0.797 & 0.0783 \\
\hline$L d(h)$ & 0.00239 & 0.00287 & 0.00269 & 0.00313 & 0.00248 & 0.00231 & 0.00254 \\
\hline$L q(h)$ & 0.00350 & 0.00386 & 0.00386 & 0.00364 & 0.00367 & 0.00367 & 0.00332 \\
\hline$V_{\text {deado }}$ & -0.0819 & -0.282 & -0.368 & -0.122 & -0.187 & -0.149 & -0.068 \\
\hline$V_{\text {deadl }}$ & -0.119 & -0.407 & -0.385 & -0.257 & -0.203 & -0.195 & -0.090 \\
\hline Mean & 1.535 & 1.562 & 1.289 & 2.43 & 2.826 & 2.690 & 1.176 \\
\hline \multirow[t]{2}{*}{ Fitness } & 0.170 & 0.383 & 0.274 & 0.554 & 0.486 & 0.486 & 0.118 \\
\hline & 6.623 & 5.305 & 1.818 & 13.603 & 19.604 & 17.988 & 0 \\
\hline
\end{tabular}

The estimation of PMSM parameters using data measured from normal temperature environment are shown in Table II , and the convergence rates of different PSOs are shown in Fig.5, the estimated PMSM parameters convergence result are shown in Fig.6(a) to Fig.6(d). The estimated VSI nonlinearities of voltage under normal temperature condition (with $i_{d}=0$ and $i_{d}=-2$ ) are shown in Fig.6(e) and Fig.6(f).It is clear from Table II that DPSO-LS provides the best performance in terms of mean, standard deviation and $t$-test values (the confidence level is 90\%). From Fig.5, the convergence speed of DPSO-LS is faster than other hybrid PSOs. The better performance of DPSO-LS can be explained in two aspects. Firstly, a novel movement modification equation using a variable exploration vector is designed to update the velocity of particles. Secondly, a dynamic $O B L$ mechanism with adaptive Gaussian distribution is introduced to overcome the blindness in the search of pBest through stochastic evolution and enables it jump out the local optimal. As demonstrated in Table II, the estimated winding resistance $(0.342 \Omega)$ with the consideration of the VSI nonlinearities is quite close to its nominal value $(0.33 \Omega)$ under normal temperature. Also, the estimated flux linkage $\psi$ $(78.3 \mathrm{mWb})$ by DPSO-LS is quite close to its nominal value $(77.6 \mathrm{mWb})$. The slight difference between the estimated and nominal values of machine parameters may be caused by nonlinearity on load condition. It is interesting that the estimated $R$ without the consideration of VSI nonlinearities is much larger than that with the consideration of VSI nonlinearities, as the influence of VSI nonlinearity distort voltages $\left(D d . V_{\text {dead }}\right.$ and $\left.D q . V_{\text {dead }}\right)$ introduce an error into the estimation of the machine parameters. The estimated distorted voltage $V_{\text {dead } 0}(i d=0)$ does not equal to $V_{\text {dead } 1}(i d \neq 0)$, and this confirms the fact that the $V_{\text {dead }}$ is directly related to the current.

As shown in Fig.6 (e) and Fig.6 (f), the value of $V_{\text {dead0 }}\left(i_{d}=0\right)$ and $V_{\text {dead1 }}\left(i_{d} \neq 0\right)$ can be estimated simultaneously with other machine parameters based on the proposed estimator model. Furthermore, the VSI nonlinearities compensation can be simultaneously obtained by computing $D d$.Vdead and adding the value of $D q$. $V$ dead to the output of $d q$-axis PI regulators. Then, the compensation on $V_{\text {dead }}$ slowly increases until $V_{\text {dead }}$ approaches to zero, and this can help reduce the its influence on system stability.

In comparison with other hybrid PSOs, the estimates of the proposed DPSO-LS are more accurate and the estimated parameters for example motor resistance, $d q$-axis inductances and the rotor flux rapidly converge to their right points. As can be seen from Fig.5, DPSO-LS converges to the optimum after about 50 generations of evolution while other hybrids shows poor convergence performance.

\section{Estimation of PMSM Parameters and VSI Nonlinearities under Varying Temperature Conditions}

In order to check the performance of the proposed method for tracking the change of parameters under varying temperature conditions, experiments on a varying temperature condition are carried out. A heater is used to heat the prototype PMSM. The temperature variation experiments are divided into two steps.

a. Continuously heating the PMSM for 20 minutes and recording experimental data.

b. Estimating the machine parameters and VSI nonlinearities of PMSM.

The comparisons of the performance of different PSOs are shown in Table III , Fig.7. the estimated PMSM parameters convergence result are shown in Fig.8(a) to Fig.8(d).The estimated VSI nonlinearities of voltage under varying temperature conditions (with $i_{d}=0$ and $i_{d}=-2$ ) are depicted in Fig.8(e) and Fig.8(f). From Table III, it is clear that DPSO-LS outperforms other hybrid PSOs in terms of mean, standard deviation and $t$-test values. From Fig.7, it can be noticed that DPSO-LS has a faster convergence speed than other hybrid PSOs. Also, the steadiness of DPSO-LS is better than the other methods. Meanwhile, as can be seen from Table III and Fig.8(a)toFig.8(d) that the estimated winding resistance $R, d$-axis inductance $L_{d}, q$-axis inductance $L_{q}$ and rotor flux linkage $\psi$ vary with the changing temperature.

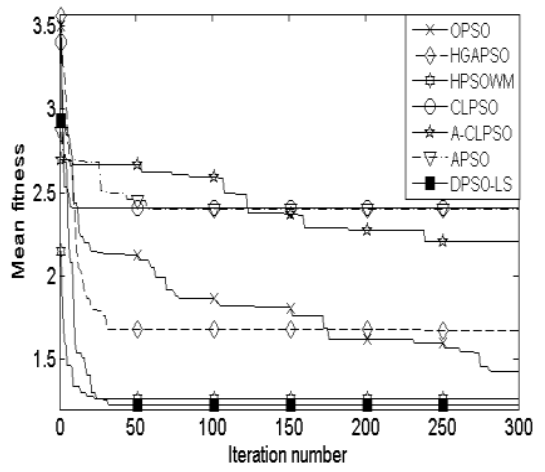

Fig. 7.The fitness convergence curve of several PSOs on PMSM parameter identification with heating 20 minutes by heater. 
TABLE III.

RESULT COMPARISONS AMONG SEVEN PSOS ON MULTIPLE PARAMETERS AND VSI NONLINEARITIES IDENTIFICATION OF PMSM UNDER

\begin{tabular}{|c|c|c|c|c|c|c|c|}
\hline Estimated Parameters & OPSO & HGAPSO & HPSOWM & CLPSO & A-CLPSO & APSO & DPSO-LS \\
\hline$R(\Omega)$ & 0.478 & 0.480 & 0.434 & 0.467 & 0.489 & 0.462 & 0.438 \\
\hline$\psi(w b)$ & 0.0765 & 0.0778 & 0.0774 & 0.0762 & 0.0759 & 0.0766 & 0.0772 \\
\hline$L d(h)$ & 0.00268 & 0.00342 & 0.00283 & 0.00202 & 0.00345 & 0.00294 & 0.00283 \\
\hline$L q(h)$ & 0.00352 & 0.0080 & 0.00393 & 0.00319 & 0.00371 & 0.00347 & 0.00329 \\
\hline$V_{\text {deado }}$ & -0.0886 & -0.361 & -0.371 & -0.173 & -0.177 & -0.098 & -0.0772 \\
\hline$V_{\text {deadl }}$ & -0.107 & -0.569 & -0.323 & -0.220 & -0.187 & -0.192 & -0.0957 \\
\hline Mean & 1.43 & 1.673 & 1.259 & 2.41 & 2.207 & 2.398 & 1.229 \\
\hline Std.dev & 0.228 & 0.702 & 0.156 & 0.624 & 0.528 & 1.02 & 0.127 \\
\hline$t$-value & 3.359 & 3.988 & 0.545 & 11.621 & 10.856 & 7.650 & 0 \\
\hline
\end{tabular}

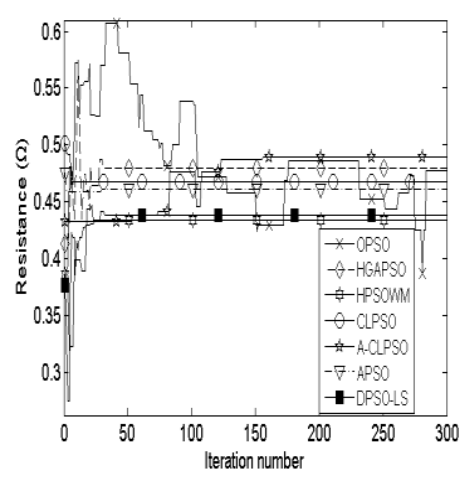

(a)

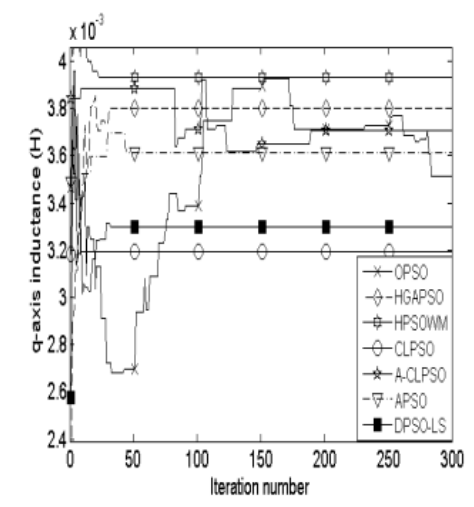

(d)

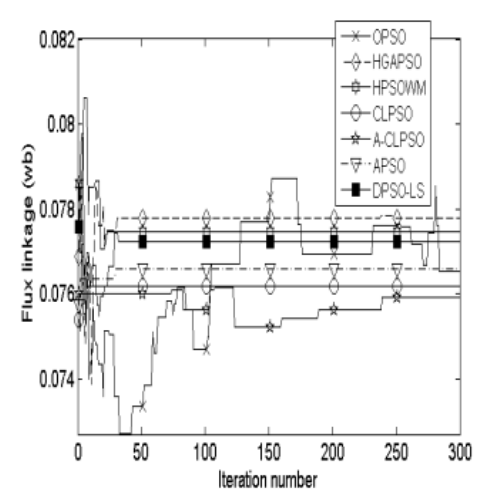

(b)

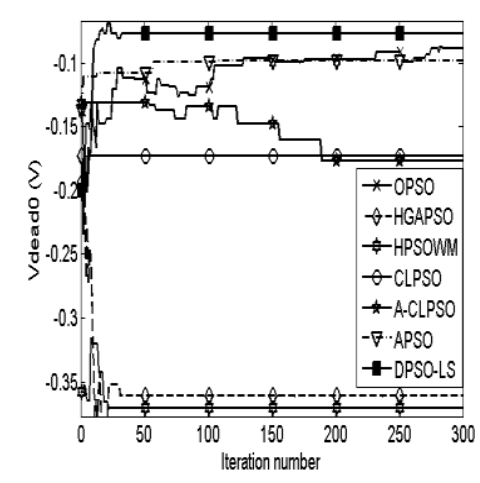

(e)

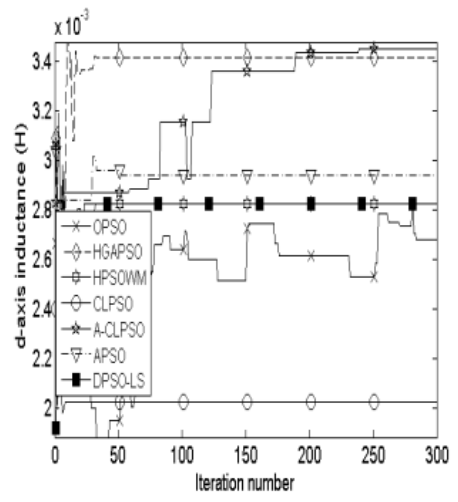

(c)

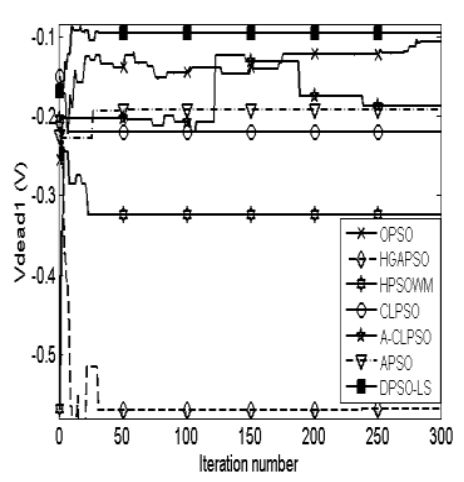

(f)

Fig. 8. Identified parameters with heating 20 minutes. (a) winding resistance.(b) rotor flux linkage.(c) $d$-axis inductance.(d) $q$-axis inductance.(e) $V_{\text {dead0 }}$.(f) $V_{\text {dead1 }}$.

For example, the estimated winding resistance value increases from $0.342(\Omega)$ to 0.438 ( $\Omega$ ) under heating temperature, the stator winding resistance value increases gradually when the temperature rises gradually due to the effects of the thermal metal. The estimated rotor flux linkage decreases from $78.3(\mathrm{mWb})$ to $77.2(\mathrm{mWb})$, the abrupt drop in the estimated rotor flux linkage after 20 minute heating can be explained by the fact that the residual flux density of the $\mathrm{PM}$ reduces when the temperature of $\mathrm{NdFeB}$ magnets increases [23].

It is interesting to note that for these PSO methods, the estimated value of $L_{\mathrm{q}}$ is larger than that of $L_{\mathrm{d}}$ in the surface-mounted PMSM. This is probably because $L_{d}$ is significantly affected by the change of permanent magnet flux and reaches a magnetic saturation status, while $L_{q}$ is not so sensitive as $L_{\mathrm{d}}$ to the change of the flux.

The estimated $L_{d}$ and $L_{q}$ also change when temperature varies, the reason is that the values of $L_{d}$ and $L_{q}$ are mainly influenced by the flux density as the flux density has changed during the data measurement after20-minitues heating. Furthermore, from Fig.6(e), Fig.6(f), Fig.8(e)and Fig.8(f), it can be seen that the estimated $V_{\text {deado }}$ varies from $-0.068(\mathrm{v})$ to $-0.072(\mathrm{v})$, the estimated $V_{\text {dead } 1}$ varies from $-0.090(\mathrm{v})$ to -0.0957 (v) after 20 minute heating, and the estimated distorted voltage $V_{\text {dead } 0}\left(i_{d}=0\right)$ does not equal to $V_{\text {dead } 1}\left(i_{d} \neq 0\right)$, this can be explained by the that the VSI nonlinearity is also influenced by the temperature variation.

The comparison of dynamic tracking performance shows that DPSO-LS is better and statistically more robust than the other hybrid PSOs in terms of global search capacity and local search precision. This may be explained that the proposed dynamic search scheme, combined with the learning strategy, play a good role in finding the global optimum for the nonlinear multimodal optimization problem here. 


\section{CONCLUSION}

A novel parameter estimation method for PMSM electrical parameters and VSI nonlinearities has been proposed, in which the estimation of the needed parameters and the influence of VSI nonlinearities were taken into account simultaneously. Compared to the conventional estimation method using the $d$ - $q$-axis equations, the proposed estimator can provide more accurate estimation of the machine parameters with the consideration of VSI nonlinearities. The VSI nonlinearities can be considered as unknown system parameters and can be simultaneously estimated using the proposed method. Furthermore, the VSI nonlinearities can be estimated individually from the $d$-q-axis equation with $i_{d}=0$ control and $i_{d} \neq 0$ (current injection condition), respectively. In order to enhance the accuracy and the dynamic performance of the estimator, a dynamic particle swarm optimization with learning strategy (DPSO-LS) is proposed to estimate the optimum parameters by minimizing the defined objective function. In the DPSO-LS, a novel movement modification equation with variable exploration vector and a Gaussian-distribution based dynamic $O B L$ operator are developed to improve search performance of particles. The proposed DPSO-LS can significantly enhance the performance of the estimator and enables it to effectively track parameter variation with the changing operation conditions. Compared with several other existing PSO algorithms, the performance of DPSO-LS performs better in tracking the variation of machine parameters and estimating the VSI nonlinearities under different operation conditions.

The proposed parameters estimation method can be adapted and applied to condition monitoring, fault diagnosis for practical industrial PMSM drive systems, such as hybrid electric vehicles, renewable energy power generation. We would also carry out further investigations on the application of the method for flux weakening mode in our future work.

\section{REFERENCES}

[1] F F M El-Sousy, "Intelligent optimal recurrent wavelet elman neural network control system for permanent-magnet synchronous motor servo drive," IEEE Trans. Ind. Informat., vol. 9,no.4 ,pp. 1986-2003,Nov. 2013. [2] J. Kim, I .Jeong, K .Lee, N. Kwanghee, "Fluctuating current control method for a PMSM along constant torque contours," IEEE Trans. Power Electron., vol.29,no.11,pp.6064-6073,Nov.2014

[3] J. Yu, P. Shi, H. Yu, B. Chen, and C. Lin, "Approximation-based discrete -time adaptive position tracking control for interior permanent magnet synchronous motors," IEEE Trans. Cybern., vol. 45, no. 7, pp. 1363-1371,Jul. 2015.

[4]K Liu, Z Q Zhu. Parameter estimation of PMSM for aiding PI regulator design of field oriented control,"2014 17th International Conference on Electrical Machines and Systems (ICEMS), IEEE, 2014, pp.2705-2711.

[5] Y Da, X Shi, M Krishnamurthy, "A new approach to fault diagnostics for permanent magnet synchronous machines using electromagnetic signature analysis," IEEE Trans. Power Electron., vol. 28, no.8, pp.4104-4112,Aug. 2013.

[6] K .Liu, Z Q Zhu, D Stone, "Parameter estimation for condition monitoring of PMSM stator winding and rotor permanent magnets," IEEE Trans. Ind. Electron., vol.60,no.12, pp. 5902-5913,Dec.2013.

[7] X. Xiao, C.M Chen, and M. Zhang, "Dynamic Permanent Magnet Flux Estimation of Permanent Magnet Synchronous Machines," IEEE Trans.App.Supercond., vol 20, no. 3, pp.1085-1088 ,Jun. 2010,

[8]K. Liu, Q. Zhang, J. Chen, Z. Zhu, and J. Zhang, "Online multiparameter estimation of nonsalient-pole PM synchronous machines with temperature variation tracking," IEEE Trans. Ind. Electron., vol. 58, no. 5, pp. 1776-1788, May ,2011.

[9]O.Sandre-Hernandez. , R.Morales-Caporal , J.Rangel-Magdaleno, $\mathrm{P}$.Heregrina-Barreto, "Parameter Identification of PMSMs Using Experimental Measurements and a PSO Algorithm," IEEE Trans. Instrum. Meas., vol. 64, no. 8,pp. 2146-2154,Aug. 2015.

[10]L. Kaiyuan, M. Vetuschi, P. O. Rasmussen, and A. E. Ritchie, "Determination of high-frequency $d$ - and $q$-axis inductances for surface-mounted permanent-magnet synchronous machines," IEEE Trans. Instrum. Meas.,vol. 59, no. 9, pp. 2376-2382, Sep. 2010.

[11]Y. Da, X. Shi, and M. Krishnamurthy, "A new approach to fault diagnostics for permanent magnet synchronous machines using electromagnetic signature analysis," IEEE Trans. Power Electron., vol. 28, no. 8, pp. 4104-4112, Aug. 2013.

[12]G.L. Wang, L.Z. Qu, H.L. Zhan, J. Xu, L. Ding, G.Q Zhang, and D.G.Xu. Self-Commissioning of Permanent Magnet Synchronous Machine Drives at Standstill Considering Inverter Nonlinearities. IEEE Trans.Power Electron., vol.29, no. 12, pp.6615-6627, Dec, 2014

[13]Q. Liu and K. Hameyer, "A fast online full parameter estimation of a PMSM with sinusoidal signal injection," in Proc. IEEE Energy Conversion Congress and Exposition (ECCE), 2015, pp. 4091-4096.

[14] G. Pellegrino, A. Vagati, P. Guglielmi, and B. Boazzo, "Performance comparison between surface-mounted and interior PM Motor drives for electric vehicle application," IEEE Trans. Ind. Electron., vol. 59, no. 2,pp. 803-811, Feb. 2012.

[15] B. N. Mobarakeh, F. Meibody-Tabar, and F.M. Sargos, "Mechanical sensorless control of PMSM with online estimation of stator resistance,"IEEE Trans. Ind. Applicat., vol. 40, no. 2, pp. 457-471, Mar.-Apr., 2004.

[16]Y. C. Shi, K. Sun, L. P. Huang, and Y. Li, "Online identification of permanent magnet flux based on extended Kalman filter for IPMSM drive with position sensorless control," IEEE Trans. Ind. Electron, vol. 59, no. 11, pp. 4169-4178, Nov. 2012.

[17] B. Thierry, L. Nicolas, N. M. Babak and M. T. Farid, "Online identification of PMSM parameters: parameter identifiability and estimator comparative study," IEEE Trans. Ind. Appl., vol. 47, no. 4, pp. 1944-1957, Jul./Aug. 2011.

[18] Y. Zhang, Z. Yin, X. Sun and Y. Zhong, "On-line identification Methods of Parameters for Permanent Magnet Synchronous Motors Based on Cascade MRAS," in Proc. 9th International Conference on Power Electronics-ECCE Asia, Seoul, Korea, pp. 345-353, Jun. 2015.

[19] S. T. Boroujeni, N. Bianchi, and L. Alberti, "Fast estimation of line-start reluctance machine parameters by finite element analysis," IEEE Trans. Energy Convers., vol. 26, no. 1, pp. 1-8, Mar. 2011.

[20]L. Liu and D. A. Cartes, "Synchronisation based adaptive parameter identification for permanent magnet synchronous motors," IET Control Theory Appl., vol. 1, no. 4, pp. 1015-1022, Jul. 2007.

[21] S. Wang, S. Shi, C. Chen, G. Yang, and Z. Qu, "Identification of PMSM based on EKF and Elman neural network," in Proc. ICAL, Aug. 2009, pp. $1459-1463$.

[22]S. D. Wilson, P. Stewart, and B. P. Taylor, "Methods of resistance estimation in permanent magnet synchronous motors for real-time thermal management," IEEE Trans. Energy Convers., vol. 25, no. 3, pp. 698-707,Sep. 2010.

[23] D. D. Reigosa, F. Briz, P. Gacia, J. M. Guerrero, and M.W. Degner, "Magnet temperature estimation in surface PM machines using high-frequency signal injection," IEEE Trans. Ind. Appl., vol. 46, no. 4, pp. 1468-1475,Aug. 2010.

[24] Kim K H, "Dual estimator-based compensation for dead time and nonlinearity unaffected by parameter variations in PMSM drive," Electronics letters, vol.48,no.23,pp. 1457-1459, Nov.2012.

[25] S. H. Hwang and J. M. Kim, "Dead time compensation method for voltage-fed PWM inverter," IEEE Trans. Energy Convers., vol. 25, no. 1, pp. $1-10$, Mar. 2010.

[26] H. W. Kim, M. J. Youn, K. Y. Cho, and H. S. Kim, "Nonlinearity estimation and compensation of PWM VSI for PMSM under resistance and flux linkage uncertainty," IEEE Trans. Control Syst. Technol., vol. 14, no. 4,pp. 589-601, Jul. 2006.

[27] K. Liu and Z. Q. Zhu, "Online estimation of rotor flux linkage and voltage source inverter nonlinearity in permanent magnet synchronous machine drives," IEEE Trans. Power Electron., vol. 29, no. 1, pp. 418-427,Jan. 2014

[28] P. Regulski, D. S. Vilchis-Rodriguez, S. Djurović, and V. Terzija, "Estimation of Composite Load Model Parameters Using an Improved Particle Swarm Optimization Method", IEEE Trans. Power Delivery, vol. 30, 
no. 2, pp.553-560,April, 2015.

[29]D.C. Huynh M.W. Dunnigan.Parameter estimation of an induction machine using advanced particle swarm optimisation algorithms. IET Electr. Power Appl., vol. 4, no. 9, pp. 748-760 ,March.2010,

[30] Whei-Min Lin,Tzu-Jung Su, and Rong-Ching Wu, "Parameter Identification of Induction Machine With a Starting No-Load Low-Voltage Test,"IEEE Trans. Ind. Electron., vol. 59, no. 1, pp.352-360,Jan ,2012

[31] Liu L, Liu WX , and Cartes D A. "Permanent magnet synchronous motor parameter identification using particle swarm optimization," International Journal of Computational Intelligence Research, vol.4,no.2,pp.211-218,Feb.2008

[32] M. Calvini, M. Carpita, A. Formentini,and M .Marchesoni, "PSO-Based Self-Commissioning of Electrical Motor Drives," IEEE Trans.Ind. Electron.,vol.62,no.2,Feb. 2015.

[33]Z. H. Liu, J. Zhang, S. W. Zhou, X. H. Li, and K. Liu, "Coevolutionary particle swarm optimization using AIS and its application in multiparameter estimation of PMSM," IEEE Trans. Cybern., vol. 43, no. 6,pp. 1921-1935. Dec. 2013.

[34] Z. H. Liu, X. H. Li, L. H. Wu, S. W. Zhou, and K. Liu, "GPU-accelerated parallel coevolutionary algorithm for parameters identification and temperature monitoring in permanent magnet synchronous machines," IEEE Trans. Ind. Informat., vol. 11, no. 5, pp. 1220-1230, Oct. 2015.

[35]S. Rahnamayan, H. R. Tizhoosh, and M. M. A. Salama, "Oppositionbased differential evolution," IEEE Trans. Evol. Comput., vol. 12, no. 1, pp. 64-79, Feb. 2008.

[36]M Ergezer, D Simon,"Mathematical and Experimental Analyses of Oppositional Algorithms," IEEE Trans. Cybern., vol.44,no.11, pp.2178-2189,Nov.2014

[37] G. E. P. Box and M. E. Muller, "A note on the generation of random normal deviates," Ann. Math. Statist., vol. 29, no. 2, pp. 610-611, Jun. 1958. [38]C. F. Juang, "A hybrid of genetic algorithm and particle swarm optimization for recurrent network design," IEEE Trans. Syst., Man, Cybern. B,Cybern, vol. 34, no. 2, pp. 997-1006, Apr. 2004.

[39] S.H .Ling, H.H.C .Iu; K.Y. Chan,H.K .Lam, B.C.W .Yeung and F.H. Leung," Hybrid Particle Swarm Optimization With Wavelet Mutation and Its Industrial Applications ", IEEE Trans. Syst., Man, Cybern. B,Cybern., vol.38,no.3, pp. 743 - 763, Jun. 2008.

[40]J. J. Liang, A. K. Qin, P. N. Suganthan, and S. Baskar, "Comprehensive learning particle swarm optimizer for global optimization of multimodal functions," IEEE Trans. Evol. Comput., vol. 10, no. 3, pp. 281-295,Jun. 2006.

[41]H. Wu.J.P Geng, R.H. Jin,J.Z. Qiu, W.Liu, J.Chen,S, Liu,” An Improved Comprehensive Learning Particle Swarm Optimization and Its Application to the Semiautomatic Design of Antennas," IEEE Trans. Antennas and Propagation.,vol.57, no.10,pp. 3018-3028, Oct. 2009.

[42] Z.H.Zhan, J. Zhang, Y. Li, and H. S.H. Chung." Adaptive Particle Swarm Optimization," IEEE Trans. Syst., Man, Cybern. B,Cybern., vol. 39, no. 6, pp.1362-1381.Dec.2009.

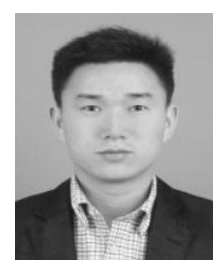

Zhao-Hua Liu (M'16) He received M.Sc. degree in computer science and engineering, and the $\mathrm{PhD}$. degree in automatic control and electrical engineering from the Hunan University, China, in 2010 and2012,respectively. $\mathrm{He}$ worked as a visiting researcher in the Department of Automatic Control and Systems Engineering at the University of Sheffield, United Kingdom, from 2015 to 2016.

$\mathrm{He}$ is currently a Lecturer with the School of Information and Electrical Engineering, Hunan University of Science and Technology, Xiangtan, China. His current research interests include evolutionary algorithms and their related applications, parameter estimation and control of permanent-magnet synchronous machine drives, and condition monitoring and fault diagnosis for power system.

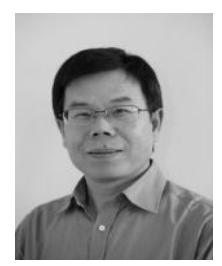

Hua-Liang Wei received the Ph.D. degrees in automatic control from The University of Sheffield, Sheffield, U.K., in 2003.

$\mathrm{He}$ is currently a senior lecturer with the Department of Automatic Control and Systems Engineering, The University of Sheffield, Sheffield, UK. His research focuses on evolutionary algorithms, identification and modelling for complex nonlinear systems, applications and developments of signal processing, system identification and data modelling to control engineering.

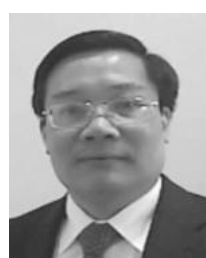

Qing-Chang Zhong (M'03-SM'04) received the Ph.D. degree in control and engineering from Shanghai Jiao Tong University, Shanghai, China, in 2000 and the $\mathrm{Ph} . \mathrm{D}$. degree in control and power engineering from Imperial College London, London, U.K., in 2004.

$\mathrm{He}$ is a Distinguished Lecturer of the IEEE Power Electronics Society and holds the Max McGraw Endowed Chair Professor in Energy and Power Engineering with the Department of Electrical and Computer Engineering, Illinois Institute of Technology, Chicago, IL, USA. the Chair Professor in Control and Systems Engineering with the Department of Automatic Control and Systems Engineering, The University of Sheffield, Sheffield, U.K. He (co)authored three research monographs, including Control of Power Inverters in Renewable Energy and Smart Grid Integration (Wiley-IEEE Press, 2013). His research focuses on power electronics and advanced control theory, together with their applications in various sectors. Dr. Zhong serves as an Associate Editor for the IEEE TRANSACTIONS ON AUTOMATIC CONTROL, IEEE TRANSACTIONS ON POWER ELECTRONICS, IEEE TRANSACTIONS ON INDUSTRIAL ELECTRONICS, IEEE TRANSACTIONS ON CONTROL SYSTEMS TECHNOLOGY, IEEE ACCESS, IEEE JOURNAL OF EMERGING AND SELECTED TOPICS IN POWER ELECTRONICS.

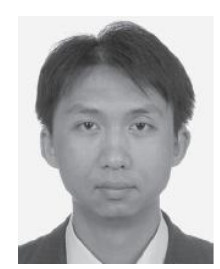

Kan Liu (M'14) received the B.Eng. and Ph.D. degrees in automation from Hunan University, Changsha,China, in 2005 and 2011 respectively. and the Ph.D. degree in electronic and electrical engineering from University of Sheffield, Sheffield, U.K., in 2013.

$\mathrm{He}$ is currently a Research Associate with the Department of Electronics and Electrical Engineering, The University of Sheffield. His research interest focuses on parameter estimation and sensorless control compensation of VSI nonlinearity. of permanent-magnet synchronous machine drives and

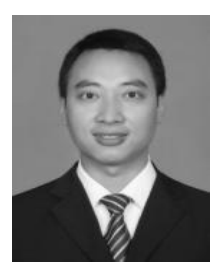

Xiao-Shi Xiao received Ph.D. degrees in Control Theory and Control Engineering from Northeastern University in 2012, respectively.

$\mathrm{He}$ is currently a Lecturer with the School of Information and Electrical Engineering, Hunan University of Science and Technology. His current research interest covers control and observer design for power systems, time-delay systems.

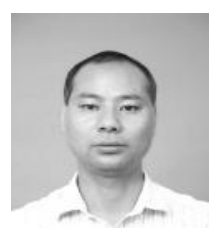

Liang-Hong Wu received the B.S. degree in industrial automation from the Hunan University of Science and Technology, Xiangtan, China, in 2001, and the M.S. and $\mathrm{Ph} . \mathrm{D}$. degrees in control science and engineering from Hunan University, Changsha, China, in 2007 and 2011, respectively.

$\mathrm{He}$ is currently an Associate Professor with the Hunan University of Science and Technology. His research power system optimal control. interests include Multi-objective optimization and 